\title{
Comparative performance of transcriptome assembly methods for non-model organisms
}

\author{
Xin Huang $^{1 *} \mathbb{D}$, Xiao-Guang Chen ${ }^{2}$ and Peter A. Armbruster ${ }^{1}$
}

\begin{abstract}
Background: The technological revolution in next-generation sequencing has brought unprecedented opportunities to study any organism of interest at the genomic or transcriptomic level. Transcriptome assembly is a crucial first step for studying the molecular basis of phenotypes of interest using RNA-Sequencing (RNA-Seq). However, the optimal strategy for assembling vast amounts of short RNA-Seq reads remains unresolved, especially for organisms without a sequenced genome. This study compared four transcriptome assembly methods, including a widely used de novo assembler (Trinity), two transcriptome re-assembly strategies utilizing proteomic and genomic resources from closely related species (reference-based re-assembly and TransPS) and a genome-guided assembler (Cufflinks).

Results: These four assembly strategies were compared using a comprehensive transcriptomic database of Aedes albopictus, for which a genome sequence has recently been completed. The quality of the various assemblies was assessed by the number of contigs generated, contig length distribution, percent paired-end read mapping, and gene model representation via BLASTX. Our results reveal that de novo assembly generates a similar number of gene models relative to genome-guided assembly with a fragmented reference, but produces the highest level of redundancy and requires the most computational power. Using a closely related reference genome to guide transcriptome assembly can generate biased contig sequences. Increasing the number of reads used in the transcriptome assembly tends to increase the redundancy within the assembly and decrease both median contig length and percent identity between contigs and reference protein sequences.

Conclusions: This study provides general guidance for transcriptome assembly of RNA-Seq data from organisms with or without a sequenced genome. The optimal transcriptome assembly strategy will depend upon the subsequent downstream analyses. However, our results emphasize the efficacy of de novo assembly, which can be as effective as genome-guided assembly when the reference genome assembly is fragmented. If a genome assembly and sufficient computational resources are available, it can be beneficial to combine de novo and genome-guided assemblies. Caution should be taken when using a closely related reference genome to guide transcriptome assembly. The quantity of read pairs used in the transcriptome assembly does not necessarily correlate with the quality of the assembly.
\end{abstract}

Keywords: Transcriptome assembly, Non-model organisms, De novo assembly, Reference-based re-assembly, TransPS, Genome-guided assembly, Next-generation sequencing, Aedes albopictus

\footnotetext{
*Correspondence: xh33@georgetown.edu

'Department of Biology, Georgetown University, 37th and O Streets NW,

Washington, DC 20057, USA

Full list of author information is available at the end of the article
} 


\section{Background}

The use of next-generation sequencing (NGS) technologies has been increasing dramatically over the past decade [1]. Due to the technological revolution in NGS, vast amounts of both transcriptome and genome sequences across a wide range of species are accumulating, especially from large-scale projects including Genome $10 \mathrm{~K}$ [2] and Insect $5 \mathrm{~K}$ [3]. Traditionally, model organisms have been chosen largely based on the ease with which they can be reared in the laboratory and used for genetic studies, or their evolutionary relatedness to human. However, in the current "-omics" era, a much greater variety of organisms can be studied at the genomic and transcriptomic level. This revolution in DNA sequencing technologies has far-reaching applications for the field of biology, dramatically increasing opportunities to elucidate gene regulatory networks [4] and the genetic basis of complex traits [5, 6]. Most importantly, NGS allows scientists to investigate biological questions at an unprecedented scale. An exceptional example is a phylogenomic analysis of the origin and diversification of major insect lineages using transcriptome sequencing and genomic data [7]. In this study, 103 species from all 33 extant insect orders have been sequenced. Combining genomic data from 41 arthropod species with transcriptomic data from 103 species, the analysis was able to resolve with high confidence the timing of the origin and diversification (topology) of insects [7], addressing a fundamental question regarding the history of life on Earth.

RNA-Sequencing (RNA-Seq) is one important NGS technology [8]. RNA-Seq samples the entire transcriptome in great depth under a particular experimental condition. The transcriptome includes all expressed sequences, which is a reduced representation of the genome. RNA-Seq has many exciting applications $[8,9]$, including the phylogenomic example mentioned above, single nucleotide polymorphism (SNP) discovery, small RNA profiling, novel transcript or splice variant discovery and comparison of global transcriptional mRNA profiles under distinct environmental conditions, e.g., benign vs. ecologically stressful conditions. RNA-Seq is a powerful tool to advance these applications for at least three reasons. First, RNA-Seq is able to capture the expression of (ideally) all genes under the specific experimental conditions. Second, it does not require any prior genetic information, which is well suited for nonmodel organisms. Third, RNA-Seq is cost-effective and affordable for most laboratories. For nearly all applications of RNA-Seq, transcriptome assembly is challenging but a crucial first step for accurate downstream genetic analyses [10].

De novo transcriptome assembly programs, which assemble short RNA-Seq reads without a reference, are the default choice for organisms without a genome sequence. De novo assembly is particularly suitable for non-model organisms, but can also be a useful assembly strategy for organisms with a genome sequence. This is because de novo assembly programs are not constrained by alignments to a reference genome and can therefore discover novel transcripts and splice variants that are not annotated in the genome [10]. However, de novo assembly is usually memory intensive, and requires high sequence coverage compared with reference-based assembly [10]. In addition, de novo methods tend to produce fragmented assemblies. Genomic resources from closely related species, including genomic scaffolds and protein sequences, can serve as a reference to guide transcriptome assembly for non-model organisms and may lead to less fragmented assemblies. Therefore, transcriptome assembly for non-model organisms can potentially benefit from genomic resources of a closely related species, as previously demonstrated [11-14]. Due to the ever-expanding pool of organisms with a sequenced genome, many researchers studying nonmodel organisms without a genome sequence but with intriguing ecological, evolutionary and public health attributes will soon find closely related genomic resources available.

Without a genome sequence for the species of interest (true for most organisms), genomic resources (protein sequences and/or genomic scaffolds) from a closely related species, if available, can serve as reference scaffolds to re-align de novo assembled contigs. For this purpose, our laboratory has developed reference-based re-assembly $[12,13]$, which is based on Scaffolding using Translation Mapping (STM) [11]. STM aligns de novo assembled contigs to protein sequences from a closely related species, and subsequently re-assembles the contigs according to the alignment results [11]. STM has been demonstrated to reduce redundancy and improve contig length relative to de novo assembly [11]. Reference-based re-assembly is modified from STM as a two-step process to incorporate both protein sequences and genomic scaffolds from a closely related species, which utilizes not only the protein coding sequences, but also genomic sequences including $5^{\prime}$ and $3^{\prime}$ untranslated regions as well as other non-coding regions $[12,13]$. Reference-based re-assembly has been shown to improve de novo assembly by increasing contig length and reducing redundancy $[12,13,15]$. Another similar approach to STM is Transcriptome Post-Scaffolding (TransPS) [14], which also utilizes protein sequences from a closely related species in order to re-assemble the de novo contigs according to how they align to the reference protein sequences. In contrast to referencebased re-assembly, TransPS merges contigs with nonoverlapping alignments to the same reference protein 
[14]. However, TransPS does not utilize genomic sequences from a closely related species. TransPS reduces the number of contigs to as few as $10 \%$ of the number of de novo assembled contigs, eliminating contigs that are redundant and increasing coverage for the reference protein sequences relative to the de novo assembly [14].

An alternative method that has been proposed to improve transcriptome assembly is genome-guided assembly, which utilizes the genome sequence of the same species as a reference to guide the transcriptome assembly [16]. If a high quality reference genome is available, this method usually generates longer contigs and is much less computationally demanding than de novo assembly [10]. With a well-annotated genome sequence, genome-guided assembly has been demonstrated to outperform de novo assembly [17]. If the genome assembly from the species of interest is not well annotated, and a well-annotated genome assembly from a closely related species is available, researchers may utilize the closely related genome to guide transcriptome assembly. However, few studies have evaluated the efficacy and accuracy of this approach (but see [18]).

The optimal strategy for transcriptome assembly will depend upon the downstream analysis and computational resources available. Data associated with extensive transcriptome sequencing in Ae. albopictus provide an outstanding opportunity to evaluate multiple methods of transcriptome assembly. Rich transcriptomic resources have been established across multiple life stages for $A e$. albopictus, including developing embryos [12], pharate larvae [13, 19] and adults [15]. Therefore, the vast majority of all the expressed genes in the genome are included in these data. Furthermore, a closely related species, Aedes aegypti, has a well-annotated genome [20], which provides protein and genomic reference scaffolds for improving de novo transcriptome assembly with reference-based re-assembly and TransPS methods. In addition, a draft genome sequence for Ae. albopictus has recently been published [21], which allows for a genome-guided assembly. Finally, the more completely assembled and annotated Ae aegypti genome assembly provides an excellent opportunity to evaluate the efficacy and accuracy of using a closely related reference genome to guide transcriptome assembly.

No study has thus far compared de novo assembly with both transcriptome re-assembly via protein reference scaffolding (i.e., reference-based re-assembly and TransPS) and genome-guided assembly simultaneously. This study utilized a comprehensive transcriptomic database of $A e$. albopictus to investigate the optimal transcriptome assembly strategy, comparing four assembly methods: a) de novo assembly using Trinity [22, 23], b) reference-based reassembly using proteomic and genomic resources from $\mathrm{Ae}$. aegypti, c) TransPS [14] using the Ae. aegypti proteome and d) genome-guided assembly by Cufflinks [24] using the recently sequenced Ae. albopictus genome and the well-annotated Ae. aegypti genome. The quality of the various assemblies was assessed by the number of contigs generated, contig length distribution, percent paired-end read mapping, and gene model representation via BLASTX [25]. We also examined differences between the gene models identified by each assembly strategy to determine whether unique subsets of gene models were identified by each method. In addition, we examined how the amount of data (number of sequence reads) affected these quality metrics of the assembly. Results from this study are particularly relevant to non-model organisms with genomic resources from a closely related species. Furthermore, the genome assembly of Ae. albopictus is fragmented due in part to its large size (1.9 Gb) and high composition (68\%) of repetitive elements [21]. However, this assembly is comparable to other published insect genomes [3], and therefore results from this study can be extended to emerging model organisms with a more fragmented genome assembly compared to traditional model organisms.

\section{Methods \\ RNA-Seq data}

Cleaned RNA-Seq paired-end reads from pooled tissue samples described in previous publications from this laboratory were used for this study. These data include reads from developing embryos [12], pharate larvae [13, 19] and adults [15] of Ae. albopictus under diapause and non-diapause conditions (read length $=101 \mathrm{bp}$ ). General procedures for generating these RNA-Seq reads from $A$. albopictus have been described previously [26]. Quality filtering of the RNA-Seq raw reads was consistent across studies and described in detail in previous publications from this laboratory [12, 13, 15]. 122,687,107 cleaned read pairs were obtained from the developing embryos, $289,860,821$ cleaned read pairs were obtained from the pharate larvae and 182,036,362 cleaned read pairs were obtained from the adult stage. In order to investigate the effect of data quantity on the quality of the assembled transcriptome, read pairs from the three life stages (embryo, larva, adult) were partitioned into three datasets containing increasing number of read pairs. We included reads from all three life stages in each dataset to maximize representation of expressed genes in the analysis. The smallest dataset, termed $180 \mathrm{M}$, was comprised of 181,225,819 read pairs, taken from one biological replicate from all experimental treatments (diapause vs. non-diapause) in each life stage. In cases where the libraries had been sequenced on different runs, the largest libraries from one lane in each run were included. The amount of data in the $180 \mathrm{M}$ dataset is the yield of approximately two lanes of Illumina HiSeq 2000 sequencing. The medium dataset, termed $360 \mathrm{M}$, 
was comprised of $344,799,731$ read pairs, taken from the remaining biological replicates of each treatment and life stage. The amount of data in the $360 \mathrm{M}$ dataset is the yield of approximately four lanes of Illumina HiSeq 2000 sequencing. The largest dataset, termed $600 \mathrm{M}$, was comprised of 594,584,290 read pairs, taken from all sequencing lanes and runs across all treatments and life stages. The amount of data in the $600 \mathrm{M}$ dataset is the yield of approximately seven lanes of Illumina HiSeq 2000 sequencing. The transcriptome assembly strategies utilized in this study and described below were applied to all three datasets (Fig. 1).
Transcriptome assembly strategies

De novo assembly

Trinity ([23]; version 20140717) was used for de novo assembly. Reads were in silico normalized with the option -normalize_max_read_cov 100 (based on recommendations in Haas et al. [23]). In addition, -min_kmer_cov 2 and -min_contig_length 200 were used to reduce memory footprint and discard contigs shorter than $200 \mathrm{bp}$, respectively. In all other respects, default parameters were used. After Trinity assembly, redundant contigs with over $99 \%$ identity were eliminated using CD-HIT-EST $[27,28]$.

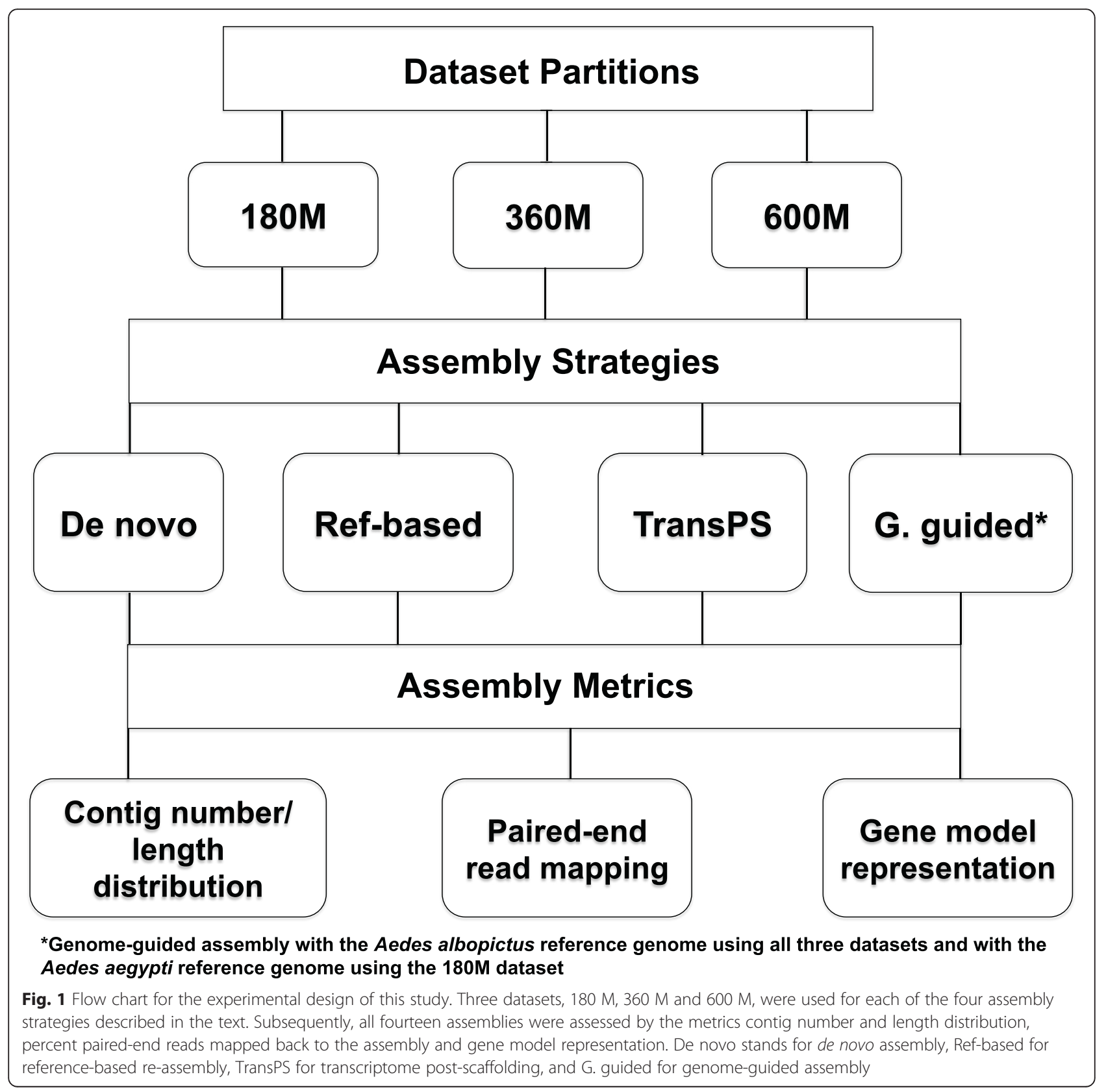




\section{Reference-based re-assembly}

After de novo assembly by Trinity, reference-based reassembly was performed as described in detail in previous publications $[12,13,15]$. The annotated Ae. aegypti protein sequences (AaegL3.3, accessed on October 8, 2014 from VectorBase [29]) were used as the protein reference for the reference-based re-assembly, in order to provide a consistent comparison with TransPS. The Ae. aegypti genome scaffolds (AaegL3) and gene annotations (AaegL3.3.gff3) used as the genomic reference were downloaded from Vectorbase [29]. Custom Perl scripts for reference-based re-assembly are available upon request.

\section{Transcriptome Post-Scaffolding (TransPS)}

After de novo assembly by Trinity, TransPS re-assembly [14] was performed using default parameters according to the authors' instructions. The Ae. aegypti proteome was selected as the scaffolding reference, because Ae. aegypti is in the same sub-genus (Stegomyia) as Ae. albopictus and the most closely related species with well-documented genomic information. Because TransPS is developed for the assembly of strand-specific RNA libraries (Z. Adelman, pers. comm.), contigs in the reverse complement orientation to the protein references were first transformed to the same orientation as the protein references based on BLASTX results. All correctly oriented contigs were then searched against the protein references using BLASTX. Contigs that matched the protein references (e-value $<1 \mathrm{e}-06)$ were reassembled by TransPS using default parameters.

\section{Genome-guided assembly - Ae. albopictus reference genome}

The genome assembly of Ae. albopictus [21] was used as the genomic reference. This assembly is comprised of $\sim 400,000$ scaffolds, with an N50 of $\sim 200 \mathrm{~kb}$ [21]. The Tuxedo suite [24], including Bowtie, Tophat and Cufflinks, was used for genome-guided assembly. For each dataset, reads from distinct life stages were first separately mapped to the genome by Tophat (-segment-length 50 -no-coverage-search), to minimize the frequencies of SNPs and computational power required [24]. Mapped reads were assembled by Cufflinks (-u -I 500000), and subsequently merged by Cuffmerge. The default parameters were used except in cases specified in parentheses above. To be consistent with the other assembly strategies, only contigs longer than 200 bp were retained. After genome-guided assembly, redundant contigs with over 99 \% identity were eliminated using CD-HIT-EST [27, 28].

\section{Genome-guided assembly - Ae. aegypti reference genome}

Using the $180 \mathrm{M}$ dataset, the genome assembly of $A e$. aegypti [20] was used as the genomic reference. This assembly is comprised of 4,757 scaffolds, with an N50 of $\sim 1.5 \mathrm{Mb}$ (AaegL3, Vectorbase). The Tuxedo suite [24], including Bowtie, Tophat and Cufflinks, was used for genome-guided assembly. Reads from distinct life stages were first separately mapped to the genome by Tophat using recommended procedures (-segment-length 50 -nocoverage-search), assembled by Cufflinks (-u -I 500000), and subsequently merged by Cuffmerge. A reference annotation file for Ae. aegypti (AaegL3.3, Vectorbase) was incorporated into Cuffmerge (-g < reference_annotation.gtf $>$ ) to guide the assemblies, as researchers would likely take advantage of the existing annotations. Cuffmerge was also performed without reference annotation. The default parameters were used except in cases specified in parentheses above. To be consistent with the other assembly strategies, only contigs longer than $200 \mathrm{bp}$ were retained. After genome-guided assembly, redundant contigs with over 99 \% identity were eliminated using CD-HIT-EST [27, 28].

\section{Comparison between genome-guided assemblies using two reference genomes}

Using the $180 \mathrm{M}$ dataset, genome-guided assemblies using the Ae. albopictus and Ae. aegypti (with reference annotation) genomes were aligned to both Ae. albopictus and Ae. aegypti genomic scaffolds via BLASTN (evalue $<1 \mathrm{e}-6$ ). Custom Perl scripts were used to calculate percent identity and contig coverage from the alignment results.

\section{Quality metrics \\ Contig number and length distribution}

Fewer and longer contigs lead to more accurate and efficient downstream read mapping. This will in turn optimize subsequent genetic analyses, such as differential gene expression analysis, SNP calling, and novel transcript or splice variant discovery. The number of contigs generated by each assembly method was recorded and the contig length distribution was represented by the median contig length. All contigs from de novo assemblies (180 M, $360 \mathrm{M}, 600 \mathrm{M}$ datasets) were aligned against the Ae. aegypti gene models with and without multiple isoforms using BLASTX (evalue $<1 \mathrm{e}-$ 6). Contigs with greater than or equal to $70 \%$ identity to the gene models and all isoforms were counted and aggregated for each dataset $(180 \mathrm{M}, 360 \mathrm{M}, 600 \mathrm{M})$. Unannotated contigs from the reference-based reassemblies were aligned to the Ae. albopictus genomic scaffolds using BLASTN (evalue $<1 \mathrm{e}-6$ ). Custom Perl scripts were used to calculate percent identity, alignment length and contig coverage.

\section{Read mapping}

A high percentage of reads mapping back to the transcriptome assembly is desirable for accurate downstream analyses, such as differential gene expression analysis, SNP calling, and novel transcript or splice variant discovery. This is because more reads mapped back to the assembly will result in increased statistical power for 
performing these analyses. Paired-end reads from each dataset and assembly method were mapped back to the corresponding assembly using Bowtie 2 ([30]; with -N 1 and insert length range specified). The percentage of total reads that mapped back and reads that uniquely mapped back to the assemblies was recorded.

\section{Gene model representation}

Measures of gene model representation described below include the number of gene models identified in the transcriptome, percent identity between the contigs and reference gene models, and percent reference coverage by the contigs.

\section{Number of gene models}

A higher number of gene models represented in the transcriptome assembly provides a basis for more comprehensive analysis of the transcriptome under a certain experimental condition. To test the representation of gene models by the transcriptome assemblies, contigs were aligned using BLASTX to the Ae. aegypti proteome (AaegL3.3). To provide a second benchmark and minimize potential bias towards reference-based reassembly and TransPS, the assemblies were also aligned using BLASTX to a non-redundant dipteran protein reference set. This dipteran protein reference set was generated by downloading orthologous protein sequences from Ae. aegypti, Culex quinquefasciatus, Anopheles gambiae and Drosophila melanogaster from OrthoDB [31], Version 7 (accessed on October 2, 2014), with one single ortholog retained per ortholog group in the order specified above (i.e., order of relatedness to Ae. albopictus). The final reference set contained 19,272 protein sequences, and represented a wide range of evolutionary diversity within Diptera with little redundancy. The best BLASTX matches with e-value smaller than 1e-6 were retained (sorted by bitscore and e-value). Custom Perl scripts were used to calculate the number of unique gene models represented in the transcriptome assemblies. In order to identify putative orthologs, reciprocal BLAST was performed for assemblies using the $180 \mathrm{M}$ dataset against the Ae. aegypti protein reference set. Assemblies were searched against the reference set using BLASTX (evalue $<1 \mathrm{e}-6$ ) and the reference set was searched against the assemblies using TBLASTN (evalue $<1 \mathrm{e}-6)$. The reference gene model identified as the best match for a contig by BLASTX was designated as a reciprocal best hit $(\mathrm{RBH})$, a potential ortholog, if the contig was identified as the best match for the gene model by TBLASTN. We didn't include contigs from the genome-guided assembly using the Ae. aegypti reference genome because of its bias towards the Ae. aegypti genomic scaffolds (see Additional file 1 and results below). We performed reciprocal BLAST for assemblies using the $180 \mathrm{M}$ dataset against the Ae. aegypti protein reference set because we have found that the $180 \mathrm{M}$ dataset is adequate for downstream transcriptome analyses (see Results below), and the Ae. aegypti and dipteran protein reference sets yield similar results (see Figs. 3 \& 4, Table 1 and Additional files 2 \& 3).

The number of gene models identified by each assembly method using the $180 \mathrm{M}$ dataset from the two reference

Table 1 Summary statistics for transcriptome assemblies from multiple strategies

\begin{tabular}{|c|c|c|c|c|c|c|c|}
\hline Assembly Strategy & $\begin{array}{l}\text { Dataset } \\
\text { used }\end{array}$ & $\begin{array}{l}\text { \# of } \\
\text { contigs }\end{array}$ & $\begin{array}{l}\text { Median } \\
\text { length (bp) }\end{array}$ & $\begin{array}{l}\text { Median \%ID } \\
\text { vs. dipteran }\end{array}$ & $\begin{array}{l}\text { Median reference } \\
\text { coverage vs. dipteran }\end{array}$ & $\begin{array}{l}\text { Median \%ID } \\
\text { vs. aegypti }\end{array}$ & $\begin{array}{l}\text { Median reference } \\
\text { coverage vs. aegypti }\end{array}$ \\
\hline \multirow[t]{3}{*}{ De novo assembly } & $180 M^{a}$ & 96,980 & 414 & $90.95 \%$ & $98.37 \%$ & $91.32 \%$ & $98.38 \%$ \\
\hline & $360 M$ & 141,390 & 394 & $90.57 \%$ & $98.21 \%$ & $91.02 \%$ & $98.14 \%$ \\
\hline & $600 \mathrm{M}$ & 176,168 & 385 & $90.43 \%$ & $98.37 \%$ & $90.97 \%$ & $98.33 \%$ \\
\hline \multirow[t]{3}{*}{ Reference-based re-assembly } & $180 \mathrm{M}$ & 89,260 & 399 & $91.04 \%$ & $98.46 \%$ & $91.45 \%$ & $98.46 \%$ \\
\hline & $360 \mathrm{M}$ & 130,168 & 382 & $90.70 \%$ & $98.22 \%$ & $91.15 \%$ & $98.26 \%$ \\
\hline & $600 \mathrm{M}$ & 162,168 & 373 & $90.57 \%$ & $98.39 \%$ & $91.10 \%$ & $98.45 \%$ \\
\hline \multirow[t]{3}{*}{ Transcriptome Post-Scaffolding } & $180 \mathrm{M}$ & 11,796 & 1,783 & $92.18 \%$ & $99.40 \%$ & $92.57 \%$ & $99.40 \%$ \\
\hline & $360 M$ & 12,027 & 1,854 & $91.97 \%$ & $99.40 \%$ & $92.41 \%$ & $99.39 \%$ \\
\hline & $600 \mathrm{M}$ & 12,117 & 1,888 & $91.90 \%$ & $99.41 \%$ & $92.39 \%$ & $99.41 \%$ \\
\hline \multirow{3}{*}{$\begin{array}{l}\text { Genome-guided assembly using } \\
\text { Ae. albopictus genome }\end{array}$} & $180 \mathrm{M}$ & 43,537 & 1,091 & $90.95 \%$ & $97.56 \%$ & $91.28 \%$ & $97.78 \%$ \\
\hline & $360 M$ & 57,524 & 1,038 & $90.73 \%$ & $97.57 \%$ & $91.05 \%$ & $97.77 \%$ \\
\hline & $600 \mathrm{M}$ & 68,977 & 1,000 & $90.56 \%$ & $97.76 \%$ & $91.03 \%$ & $97.93 \%$ \\
\hline $\begin{array}{l}\text { Genome-guided assembly using } \\
\text { Ae. aegypti genome }+\mathrm{RA}^{\mathrm{b}}\end{array}$ & $180 \mathrm{M}$ & 18,999 & 1,426 & $100.00 \%$ & $100.00 \%$ & $100.00 \%$ & $100.00 \%$ \\
\hline $\begin{array}{l}\text { Genome-guided assembly using } \\
\text { Ae. aegypti genome-RA }\end{array}$ & $180 \mathrm{M}$ & 13,230 & 348 & $100.00 \%$ & $42.47 \%$ & $100.00 \%$ & $42.89 \%$ \\
\hline
\end{tabular}

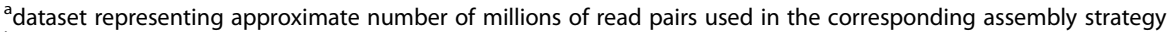

${ }^{\mathrm{b}} \mathrm{RA}$ refers to reference annotation 
protein sets was visualized as quadruple Venn diagrams, constructed by the VennDiagram package in the R software environment (www.r-project.org). Genome-guided assembly using the Ae. aegypti reference genome was not included in this analysis because of the above-mentioned bias. The $360 \mathrm{M}$ and $600 \mathrm{M}$ datasets were not used in this analysis because each assembly method generated similar numbers of gene models across all three datasets $(180 \mathrm{M}$, $360 \mathrm{M}, 600 \mathrm{M}$ ) for each of the two reference protein sets (see Results below). The unique gene models identified by de novo and genome-guided assemblies were tested for enrichment of specific Gene Ontology (GO) terms and KEGG pathways using the Bioconductor GOseq package [32] in R. GO terms for the dipteran gene models were obtained from Ensembl Metazoa [33]. KEGG pathway assignments for the dipteran organisms in the two reference protein sets were obtained from KEGG API [34]. Because GO terms and KEGG pathways only correspond to genes rather than specific isoforms, isoforms were consolidated within each gene model in the enrichment analyses.

\section{Percent identity}

A higher median percent identity (PID) indicates higher confidence in annotating the Ae. albopictus contigs as putative orthologs of the reference gene models, which is important for interpreting downstream analyses. Custom Perl scripts were used to calculate the PID between contigs and their best BLASTX matches.

\section{Reference coverage}

A high coverage of the reference gene models by assembled contigs indicates that the annotated gene models are well represented in the Ae. albopictus transcriptome, which leads to high confidence in accurate subsequent genetic analyses. Custom Perl scripts were used to calculate the percent coverage for the protein reference in each match (i.e., the proportion of the reference represented in a match).

\section{Results}

Due to the substantial computational requirements of performing transcriptome assembly with some of the methods considered in this study and the large RNA-Seq datasets we analzyed, it was only feasible to perform one assembly for each method and dataset. Therefore, we discuss the absolute magnitude of clear differences and/or high similarities between the assembly methods and between datasets $(180 \mathrm{M}, 360 \mathrm{M}, 600 \mathrm{M})$ within assembly methods.

\section{Contig number}

Consistently across all three datasets, de novo assembly produced the largest numbers of contigs, followed closely by reference-based re-assembly. Genome-guided assembly using the Ae. albopictus reference genome produced less than half the number of contigs produced by de novo assembly. Genome-guided assembly using the $180 \mathrm{M}$ dataset and the Ae. aegypti genome produced similar number of contigs relative to TransPS, but fewer than other assembly methods (Table 1). TransPS produced the smallest numbers of contigs, with over $87 \%$ reduction in the number of contigs relative to de novo assembly (Table 1). Consistently across all assembly strategies performed using three datasets, increasing the amount of input read pairs used in the transcriptome assembly led to an almost two-fold increase in the number of contigs generated, except for TransPS, where the increase was minimal (Table 1). The number of contigs aligned to annotated gene models and isoforms did not increase substantially with increasing number of read pairs in the datasets (see Additional file 4). More than $84 \%$ of the unannotated contigs aligned to the Ae. albopictus genomic scaffolds, with high median percent identity ( $93 \%)$ and contig coverage $(>97 \%)$ by the genomic scaffolds, though with short median alignment length (255-258 bp, see Additional file 4).

\section{Contig length distribution}

With reference annotation, genome-guided assembly using the Ae. aegypti genome generated contigs longer than any other assembly method, except for TransPS (Table 1). Without reference annotation, genome-guided assembly using the Ae. aegypti reference genome generated the shortest contigs (Table 1). Consistently across three datasets among all other assembly methods, de novo assembly and reference-based re-assembly generated similar and the smallest median contig lengths. Genomeguided assembly using the Ae albopictus reference genome generated median contig lengths more than twice as long as those by de novo assembly and reference-based re-assembly. Finally, the TransPS assembly generated the greatest median contig length with a more than four-fold increase relative to de novo assembly and reference-based re-assembly (Table 1). Within each assembly strategy performed using three datasets, increasing the amount of read pairs used in the transcriptome assembly slightly decreased the median contig length, with the exception of TransPS, which generated slightly greater median contig lengths with more input read pairs (Table 1).

\section{Paired-end read mapping}

Consistently across all three datasets, de novo assembly, reference-based re-assembly and genome-guided assembly using the Ae. albopictus reference genome produced a similar percentage of paired-end reads mapped back, ranging from 84.73 to $93.06 \%$ (Fig. 2). Less than $80 \%$ of the paired-end reads mapped back to the TransPS 


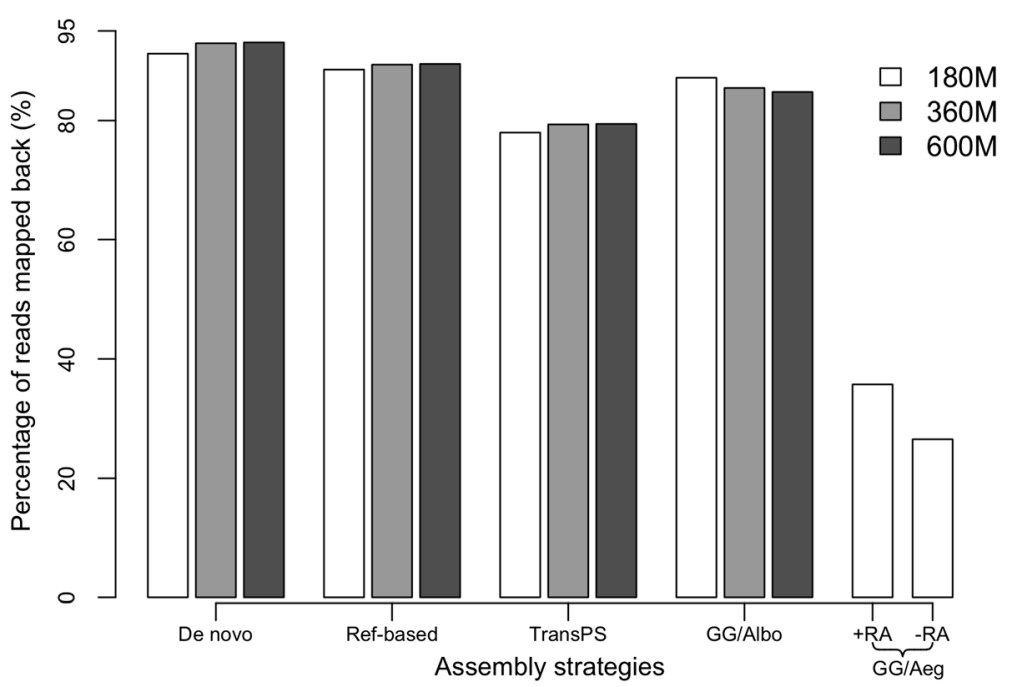

Fig. 2 Percentage of paired-end reads mapping back to the assembly. Datasets (180 M, 360 M, 600 M) and assembly strategies as described in Fig. 1, GG/Albo refers to genome-guided assembly using the Ae. albopictus reference genome and GG/Aeg refers to genome-guided assembly using the Ae. aegypti reference genome. +RA refers to genome-guided assembly using the Ae. aegypti genome with reference annotation, and -RA refers to genome-guided assembly using the Ae. aegypti genome without reference annotation

assemblies (Fig. 2). Genome-guided assembly using the Ae. aegypti reference genome had the lowest percentage of reads mapped back (<40\%, Fig. 2). When considering uniquely mapped reads, de novo assembly and referencebased re-assembly had a similar percentage of reads uniquely mapped back and TransPS had a slightly higher percentage of reads uniquely mapped back (all above $70 \%$, see Additional file 5). Genome-guided assembly using the Ae. albopictus reference genome had a much lower percentage of reads uniquely mapped back $(<50 \%)$ and genome-guided assembly using the Ae. aegypti reference genome had the lowest percentage of reads uniquely mapped back $(<8 \%$, see Additional file 5 ). Within each assembly strategy performed using three datasets, increasing the amount of read pairs used in the transcriptome assembly did not have a large or consistent effect on the read mapping percentage.

\section{Number of gene models}

For all datasets, most assemblies produced similar numbers of gene models from both Ae. aegypti and dipteran protein reference sets (see Fig. 3 and Additional file 2), with the number of dipteran gene models generated by TransPS assemblies slightly lower than the other methods $(\sim 8,500$ vs. $>9,100)$. The exceptions were that genome-guided assembly using the Ae. aegypti reference genome with reference annotation produced the highest numbers of both Ae. aegypti and dipteran gene models and that genome-guided assembly using the Ae. aegypti reference genome without reference annotation produced the lowest numbers of both Ae. aegypti and dipteran gene models (see Fig. 3 and Additional file 2).
Within each assembly strategy performed using three datasets, increasing the amount of input read pairs led to a very slight increase in the number of Ae. aegypti gene models represented in the assembly ( 200, Fig. 3), although increased input read pairs did not have an apparent effect on the number of dipteran gene models represented in the assembly (see Additional file 2). The number of gene models discovered from the dipteran protein reference set was smaller than that from the $A e$. aegypti protein reference set, which is expected due to the closer relationship of Ae. albopictus to Ae. aegypti relative to the other species in the dipteran protein reference set. Using the $180 \mathrm{M}$ dataset, TransPS generated the highest percentage of RBHs, and the largest number of potential orthologs, amongst all assembly methods, the rest of which performed similarly (see Additional file 6). Genome-guided assembly using the Ae. albopictus reference genome had the highest median alignment length when searched against the Ae. aegypti protein reference set (see Additional file 6). The protein reference set had the highest median alignment length when searched against the TransPS assembly (see Additional file 6).

Using the $180 \mathrm{M}$ dataset, reference-based re-assembly and TransPS identified the same subsets of gene models identified by de novo assembly, with very few exceptions (see Fig. 4 and Additional file 3). This result is expected because these two methods are both re-assembly strategies performed after de novo assembly. Also using the $180 \mathrm{M}$ dataset, the de novo assembly and genome-guided assembly using the Ae. albopictus reference genome identified 1,375 and 988 unique gene models relative to each other from the Ae. aegypti reference protein set, respectively (Fig. 4). 


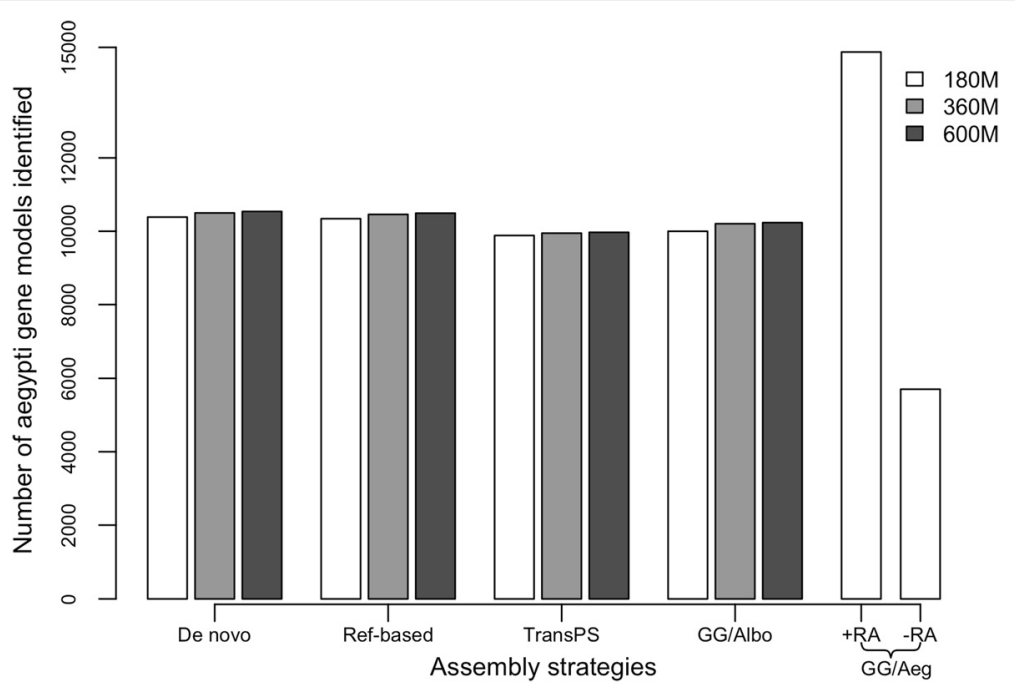

Fig. 3 Number of gene models identified from the Ae. aegypti reference protein set in all assemblies. Datasets and assembly strategies as in Fig. 2

Finally, the de novo assembly and genome-guided assembly using the Ae. albopictus reference genome identified 1,036 and 935 unique gene models relative to each other from the dipteran reference protein set, respectively, using the $180 \mathrm{M}$ dataset (see Additional file 3). In all cases, the unique gene models were not enriched for specific GO terms or KEGG pathways (all FDR-corrected $p$ values are greater than or equal to 0.11 ).

\section{Percent identity}

Genome-guided assembly using the Ae. aegypti reference genome generated contigs with the perfect $(100 \%)$ median percent identity (PID) to corresponding Ae. aegypti and dipteran gene models (Table 1). Consistently across three datasets among all other assembly methods, TransPS

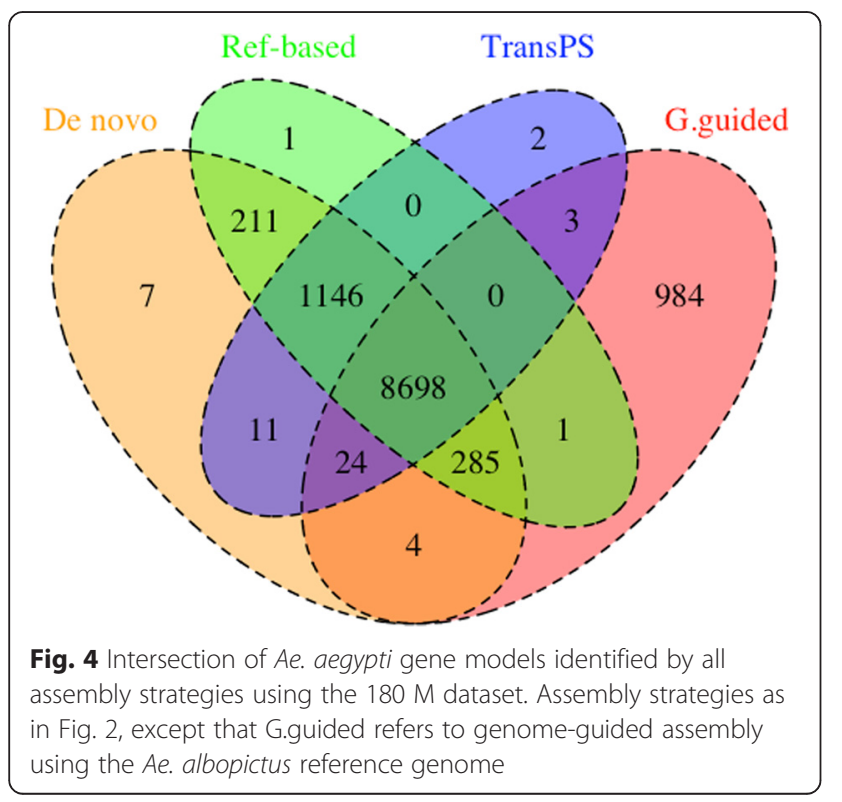

generated contigs with the highest median PID to corresponding Ae. aegypti and dipteran gene models, followed closely by reference-based re-assembly, de novo assembly and genome-guided assembly using the Ae. albopictus reference genome, the latter three with similar PID values (Table 1). Within each assembly strategy performed using three datasets, increasing the amount of read pairs used in the transcriptome assembly decreased median PID to both Ae. aegypti and dipteran gene models.

\section{Reference coverage}

Median reference coverage by Ae. albopictus contigs for both Ae. aegypti and dipteran gene models was high (most above $97.50 \%$ ) and similar across most transcriptome assembly strategies using each dataset (Table 1). Genome-guided assembly using the Ae. aegypti reference genome with reference annotation produced the highest median reference coverage (100\%). The exception was that genome-guided assembly using the Ae. aegypti reference genome without reference annotation produced the lowest median reference coverage (below $50 \%$ ).

\section{Genome-guided assembly - Ae. albopictus genome versus Ae. aegypti genome}

Contigs from the genome-guided assembly using the $A e$. albopictus reference genome resembled the Ae. albopictus genomic scaffolds (median percent identity $=100 \%$, median contig coverage $=100 \%$ ), rather than the $A e$. aegypti genomic scaffolds (median percent identity= $78.34 \%$, median contig coverage $=76.02 \%$, see Additional file 1A). On the contrary, with reference annotation, contigs from the genome-guided assembly using the $A e$. aegypti reference genome resembled the Ae aegypti genomic scaffolds (median percent identity $=100 \%$, median contig coverage $=100 \%$ ), rather than the Ae. 
albopictus genomic scaffolds (median percent identity = $80.68 \%$, median contig coverage $=86.90 \%$, see Additional file 1B). This result likely indicates the presence of ancestral polymorphism in the Ae. albopictus genome after 71.4 million years of divergence from Ae aegypti [21] and emphasizes the limitation of using a closely related reference genome for guiding transcriptome assembly with the Tuxedo suite workflow.

\section{Discussion}

Transcriptome assembly is a crucial first step for transcriptome analyses using RNA-Seq data. With the rapid development in the NGS technologies, genomic and transcriptomic resources have accumulated rapidly for a wide range of organisms. However, deciding on the optimal transcriptome assembly strategy for non-model organisms remains a challenge, especially with the large amount of data generated by NGS. This study is the first to compare de novo assembly with genome-guided assembly, reference-based re-assembly and TransPS, the latter two methods representing transcriptome re-assembly using protein sequences from closely related species. This study is also one of the first to compare genome-guided assembly using a reference genome from the focal study organism and a reference genome from a closely related species. Our analysis takes advantage of three datasets with increasing numbers of Illumina paired-end reads.

A previous study [35] used genomic contigs from the same species or genomic scaffolds from a closely related species (90-95\% identity) as a scaffold to re-assemble de novo contigs generated from RNA-Seq reads. Results from this study [35] showed that genomic sequences from the same species or closely related species can improve the representation of full-length reference transcripts by the de novo transcriptome assembly. However, high coverage genomic sequences are often not available together with RNA-Seq reads, as high coverage whole genome sequencing is still expensive, especially for species with a large genome. In addition, genomic resources from closely related species with higher than $90 \%$ identity are relatively rare. In contrast, this study used protein sequences from a more distantly related species (Ae. aegypti, in the same sub-genus, Stegomyia, as Ae. albopictus) as the reference scaffolds. This study also considered genomic scaffolds from Ae. aegypti with $80-85 \%$ identity at the nucleotide level relative to the comparison noted above. Thus, both re-assembly approaches used in this study (reference-based re-assembly and TransPS) are likely to apply to a wide range of organisms. Although the optimal assembly strategy will depend upon the purpose and downstream analyses of the specific study, our analysis provides general guidance that is likely to be useful to researchers conducting transcriptome analyses in non-model organisms.

\section{De novo assembly - Trinity}

Trinity, which has been optimized for runtime performance [36], has been demonstrated to be an effective de novo transcriptome assembler [23, 37, 38]. Trinity de novo assembly performed similarly to genome-guided assembly using the Ae. albopictus reference genome in terms of percent read mapping back to the assembly and gene model representation, though not in terms of contig number or length distribution. One of the advantages of de novo assembly is that gene models can be assembled that may not be assembled by a genome-guided method if the reads cannot be mapped back to the current genomic scaffolds due to improper scaffold assembly (see Fig. 4 and Additional file 3). It is straightforward and fast (24-48 h) to perform the Trinity assembly pipeline using high quality RNA-Seq reads, though it typically requires a large amount of memory (up to 256GB of RAM in this study). Thus, Trinity will usually require a high performance computing cluster or the cloud environment. Trinity incorporates all reads simultaneously without the need to separately perform assemblies of reads from different life stages or environmental conditions. The assemblies generated by the Trinity de novo method had high read mapping percentage, and represented a substantial number of gene models with high median PID and coverage. Identifying more total gene models, including paralogs, can be more beneficial for downstream differential gene expression and enrichment analyses, because most paralogs have similar biological functions and are included in gene ontology and KEGG pathways. Trinity assemblies had slightly lower unique mapping rates than TransPS assemblies, but because read mapping programs, such as RSEM [39], take into account reads mapped to multiple gene models, total read mapping rates are more informative than unique mapping rates for most downstream analyses. Therefore, the Trinity assemblies would be suitable for subsequent differential gene expression analysis, SNP discovery in coding regions, and also novel transcript or splice variant discovery. However, the Trinity assemblies contain more redundancy relative to assemblies generated by genome-guided and TransPS methods. Redundant contigs represent highly similar sequences corresponding to the same reference and are in part due to the fact that RNA-Seq was performed on mRNAs from tissue samples that were pooled from multiple individuals, a common practice in RNA-Seq studies. The increased redundancy of the Trinity assemblies is reflected in the larger number of contigs, but similar number and coverage of reference gene models relative to the other assembly methods. The redundancy within the de novo assembly increased with increasing amount of input read pairs, primarily due to the increase in unannotated rather than annotated contigs (see Additional 
file 4). These unannotated contigs align to unannotated protein coding sequences and non-coding sequences, including $5^{\prime}$ and $3^{\prime}$ untranslated regions.

\section{Reference-based re-assembly}

Our previous study [15] has demonstrated that referencebased re-assembly can improve de novo assembly in terms of contig number and length distribution. However, in the current study, reference-based re-assembly performed similarly to de novo assembly using Trinity (Table 1). There are two likely reasons for this result. First, different versions of Trinity were used in the previous and the current studies, and the latter is an improved version. Second, our previous study only used short RNA-Seq reads from the adult stage for the de novo assembly, which were then combined with previous contigs from other life stages in a reference-based re-assembly, including contigs assembled from 454 sequencing reads. However, the current study assembled short Illumina RNA-Seq reads from all life stages into a de novo assembly. The contigs from this de novo assembly were then used in the reference-based re-assembly, but were not combined with any previously assembled contigs. The advantage of reference-based re-assembly is that it can combine contigs from previous studies under different environmental conditions using different sequencing platforms [12, 13, 15], which makes the re-assembly less computationally demanding and more flexible. Furthermore, referencebased re-assembly can incorporate both protein and genomic sequences from closely related species, maximizing the information provided by reference scaffolding. However, reference-based re-assembly is more complicated to perform than the straightforward Trinity de novo assembly pipeline because it involves using several custom Perl scripts for the re-assembly, generally taking approximately 2 weeks to complete after de novo assembly.

\section{TransPS}

In the current study, we used a reference proteome from Ae. aegypti. As mentioned above, Ae aegypti is in the same sub-genus (Stegomyia) as Ae. albopictus. This is similar to the level of divergence between one of the organisms (Ixodes ricinus) and its reference (I. scapularis) used in the original study [14]. TransPS reduced the number of contigs by more than $87 \%$, a result similar to the original description of this method [14]. TransPS also produced the longest contigs with more than a four-fold increase in the median contig length relative to de novo assembly and reference-based reassembly (Table 1), with minimal redundancy. Thus, the TransPS assemblies had the lowest number of contigs per gene model among all four assembly methods. Moreover, the median coverage for reference gene models by TransPS assemblies was the highest amongst all methods (>99 \%), except for the genome-guided assembly using the Ae. aegypti genome with reference annotation (Table 1), indicating that TransPS assemblies had almost complete representation for the reference coding sequences. TransPS assembly also had the highest percentage of reciprocal best hits (RBHs, see Additional file 6). Out of 17,158 Ae. aegypti gene models, 3,952 are at least $70 \%$ identical to other gene models (not isoforms), with median percent identity of $97.88 \%$ and median coverage of $99.65 \%$. Because TransPS generated the longest contigs (Table 1) by also merging contigs with non-overlapping alignments to the same gene model, the TransPS contigs can better distinguish small differences between highly similar gene models, thereby identifying the most RBHs (see Additional file 6). This advantage is reflected in the longest median alignment length when searching the reference set against the assemblies (TBLASTN), not when searching the assemblies against the reference set (BLASTX, see Additional file 6). However, the overall number of gene models and read mapping percentage of the TransPS assemblies were slightly lower than the other three strategies, which could limit some downstream analyses such as differential gene expression analysis, SNP discovery, or novel transcript and splice variant discovery. This result is likely due to the algorithm used by TransPS to re-assemble the de novo assembly via protein sequences from a closely related species, without the untranslated regions or non-coding sequences in the genomic scaffolds utilized by the reference-based reassembly or genome-guided assembly. On the other hand, because TransPS had the highest percentage of reads uniquely mapped back, which enables accurate identification of orthologs, TransPS may be preferable for other downstream analyses, such as phylogenomic studies. A few thousand complete and orthologous genes from each transcriptome will be sufficient to construct a phylogeny with high confidence (e.g., [7]). Identifying the largest number of potential orthologs via reciprocal BLAST further strengthens the advantage of using TransPS for phylogenomic studies. As described in the Methods section, TransPS is developed specifically for assembly of strandspecific libraries, and therefore extra steps are required if the initial assembly (prior to scaffold re-assembly) is generated from non-strand-specific sequencing reads. While the TransPS script took an hour to finish, generating the necessary BLASTX outputs and the extra steps necessary to handle non-strand-specific sequencing reads took approximately 2 weeks to complete.

\section{Genome-guided assembly - Cufflinks: Ae. albopictus reference genome}

The number and reference coverage of gene models generated by the genome-guided method using the Ae. 
albopictus reference genome were very similar to those produced by de novo assembly and reference-based reassembly. However, genome-guided assemblies using the Ae. albopictus reference genome produced less than half of the number of contigs generated by de novo assembly and reference-based re-assembly (Table 1), indicating that this assembly produced lower redundancy relative to these two methods. In addition, genome-guided assemblies using the Ae. albopictus reference genome produced median contig lengths more than twice as long as those produced by de novo assembly and referencebased re-assembly (Table 1). Furthermore, genomeguided assembly is much less computationally demanding than de novo assembly. Genome-guided assembly using Cufflinks requires the most computational resources during read mapping to the genome. In this study, up to $72 \mathrm{~GB}$ of RAM was used for the read mapping using TopHat and up to 36GB of RAM was used for the Cufflinks genome-guided assembly. It took approximately 1 week to complete read mapping and assembly using the pipeline. Another advantage of genome-guided assembly is that transcripts with low sequencing coverage can be assembled if the reads can be mapped to the genomic scaffolds, which can lead to unique gene models assembled relative to de novo assembly (see Fig. 4 and Additional file 3). However, genome-guided assembly using the Ae. albopictus reference genome performed similarly to those generated by de novo assembly and reference-based re-assembly in terms of percent read mapping back to the assembly and gene model representation, and had the lowest unique mapping rates. This is likely because of the fact that the current Ae. albopictus genome assembly is relatively fragmented, largely due to its huge genome size and high composition of repetitive elements in the genome [21]. However, the fragmented assembly of the Ae. albopictus genome ( 400,000 scaffolds with an N50 of $\sim 200 \mathrm{~kb})$ is similar to other insect genomes. For example, the sand fly genome, Phlebotomus papatasi, has 106,826 scaffolds with an N50 of 27,956 bp [3]. Therefore, our current results are likely relevant to a wide range of organisms with similarly fragmented genome assemblies. Genomeguided assemblies can be suitable for differential gene expression analysis and SNP discovery, but may be less desirable for novel transcript and splice variant discovery, because only reads aligned to the known splice junctions can be assembled.

\section{Genome-guided assembly - Cufflinks: Ae. aegypti reference genome}

Ae. aegypti is a closely related species in the same subgenus (Stegomyia) as Ae. albopictus. Ae. aegypti has wellannotated genomic resources and a much less fragmented genome assembly, which helps to identify more complete gene models (see Fig. 3 and Additional file 2). However, using the Ae. aegypti rather than the more fragmented Ae. albopictus genome to guide transcriptome assembly can be problematic. Genome-guided assembly using the Ae. aegypti reference genome produced contigs that resemble the Ae. aegypti genomic scaffolds, rather than the Ae. albopictus genomic scaffolds (see Additional file 1). This result is reflected by the low percentage of reads mapped back, perfect median percent identity and reference coverage values of contigs from the genome-guided assembly using the Ae. aegypti genome with reference annotation (see Table 1 and Additional file 5).

\section{Combination of de novo and genome-guided assemblies}

Assemblies generated by the de novo and genome-guided methods identified a small number of unique gene models relative to each other, which is consistent with a previous study [40]. Therefore, it might be beneficial to combine genome-guided and de novo assemblies, as proposed previously [10]. When the genome reference is not well assembled, de novo assembly should be performed first, followed by aligning contigs from the de novo assembly to the genome reference in order to extend and scaffold the contigs [10]. However, if the annotation of the genome is not complete, additional annotation based on orthologs will still be necessary. As a proof of concept, we combined de novo assembly and genome-guided assembly using the $A e$. albopictus reference genome for the $180 \mathrm{M}$ dataset. The combined assembly encompassed almost all Ae. aegypti and dipteran gene models from the two separate assemblies (see Fig. 4 and Additional file 3). This result indicates that combining the de novo and genome-guided assemblies can generate a more comprehensive transcriptome assembly in terms of number of gene models identified.

\section{Effect of the number of reads on assembly quality}

The quantity of input read pairs increased the redundancy within the assembly, and decreased both median contig length and PID, whereas it did not have a clear effect on other quality metrics, i.e., percent read mapping and gene model representation. This is likely due to the increasing number of mismatches in the larger datasets caused by SNPs from the pooled tissue samples and/or sequencing errors. These mismatches caused difficulties for the assembly programs to consolidate highly similar contigs. This in turn increased the number of contigs generated (increased redundancy with similar number of gene models and reference coverage), and reduced both the median contig length and PID. Therefore, it will generally be beneficial and cost-effective to use fewer reads (i.e., the $180 \mathrm{M}$ dataset). The exception was TransPS, which generated slightly longer contigs with more input read pairs (Table 1). This is likely because the algorithm used in TransPS that assembles contigs that have non- 
overlapping matches to the same reference, thereby creating longer contigs [14].

\section{Conclusions}

Our results emphasize the efficacy of de novo transcriptome assembly using high quality Illumina RNA-Seq reads and the importance of genome assembly and annotation for genome-guided assembly. Despite generating a more fragmented transcriptome assembly, de novo assembly performed similarly to genome-guided assembly using the Ae. albopictus reference genome in terms of read mapping and gene model representation. With high quality reads, de novo assembly can prove to be effective for organisms without a genome sequence, or for organisms with a fragmented genome assembly relative to traditional model organisms. A recent study points out that even with well curated human and worm genomes, de novo assembly still performs similarly to genome-guided assembly in terms of sensitivity in constructing the transcriptome using simulated data [41]. Previous studies have demonstrated a better performance by genome-guided assembly than de novo assembly $[17,35]$. However, this study showed that, if the genome assembly is fragmented and/or genome annotation is incomplete, de novo assembly can perform similarly to or even outperform genome-guided assembly in terms of read mapping back to the assembly and gene model representation. Researchers need to be cautious when using a closely related reference genome to guide transcriptome assembly, because the resulting assembly can be biased towards the closely related genome rather than the focal genome. In our analysis, this result occurred when using a reference genome from the same subgenus with approximately 70 million years of divergence (see Additional file 1). Reference-based re-assembly performed similarly to de novo assembly, whereas TransPS generated the longest contigs with higher reference coverage, least redundancy and largest number of potential orthologs (RBHs). However, TransPS also produced assemblies with the lowest percent read mapping and number of gene models identified. Our results also reveal that the amount of input read pairs tended to reduce the quality of the resulting transcriptome assembly. Thus, $180 \mathrm{M}$ high quality paired-end reads will usually be sufficient to generate a transcriptome assembly appropriate for downstream analyses. The optimal transcriptome assembly strategy is dependent upon intended downstream analyses, but in general, Trinity de novo assembly with $180 \mathrm{M}$ high quality read pairs is suitable for most downstream transcriptome analyses, especially for organisms without a genome sequence. If a genome assembly is present, it can be beneficial to combine de novo and genome-guided assemblies when the computational resources are available.

\section{Additional files}

\begin{abstract}
Additional file 1: Similarity of genome-guided assemblies using the $180 \mathrm{M}$ dataset to reference genome assemblies. Percent identity to and contig coverage by the Aedes albopictus or Aedes aegypti genomic scaffolds characterized by BLASTN searches (evalue < 1e-6). A: Comparisons between genome-guided assembly using the Ae. albopictus genome assembly or the Ae. aegypti genome assembly and the Ae. albopictus genomic scaffolds. B: Comparisons between genome-guided assembly using the Ae. albopictus genome assembly or the Ae. aegypti genome assembly and the Ae. aegpyti genomic scaffolds. Albopictus G.guided: genome-guided assembly using the Ae. albopictus genome; Aegypti G.guided: genome-guided assembly using the Ae. aegypti genome with reference annotation; Albopictus scaffolds: genomic scaffolds of Ae. albopictus; Aegypti scaffolds: genomic
\end{abstract} scaffolds of Ae. aegypti. (PDF 6 kb)

Additional file 2: Number of gene models identified from the dipteran reference protein set in all assemblies. Datasets and assembly strategies as in Fig. 2. (TIF 8440 kb)

Additional file 3: Intersection of dipteran gene models identified by all four assembly strategies using the $180 \mathrm{M}$ dataset. Assembly strategies as in Fig. 2, except that G.guided refers to genome-guided assembly using the Ae. albopictus reference genome. (TIFF $902 \mathrm{~kb}$ )

Additional file 4: Alignment to Ae. aegypti gene models and $A e$. albopictus genomic scaffolds by contigs in the De novo assemblies and reference-based re-assemblies with increased number of input read pairs. For De novo assemblies: dataset used, number of contigs, the total number of contigs supporting Ae. aegypti gene models with multiple isoforms, the total number of contigs supporting Ae. aegypti gene models with a single isoform and the total number of contigs supporting all isoforms. Support is characterized as the best blastx match (evalue $<1$ e-6) with percent identity $>=70 \%$. For unannotated contigs from the reference-based re-assemblies: dataset used, number of contigs, total number of contigs aligning to the Ae. albopictus genome (blastn with evalue < 1e-06), median percent identity of the matches, and median alignment length with the median contig coverage in parentheses. (XLSX 40 kb)

Additional file 5: Total and unique read mapping percentages. Dataset used in, percentage of total reads and percentage of reads uniquely mapped back to the transcriptome assemblies generated in this study. (XLSX 34 kb)

Additional file 6: Number and percentage of gene models identified by reciprocal BLAST and median alignment length. For various transcriptome assembly methods: Dataset used, number of Ae. aegypti gene models identified by BLASTX, percentage of Ae. aegypti gene models identified by reciprocal blast (BLASTX and TBLASTN), median alignment length from BLASTX and TBLASTN. All BLAST matches have e-value $<1 \mathrm{e}-6$ and percent identity $>=70 \%$. (XLSX $30 \mathrm{~kb})$

\section{Abbreviations}

GO, Gene Ontology; NGS, Next-generation Sequencing; PID, Percent Identity; $\mathrm{RBH}$, Reciprocal Best Hit; RNA-Seq, RNA-Sequencing; SNP, Single Nucleotide Polymorphism; STM, Scaffolding using Translation Mapping; TransPS, Transcriptome Post-Scaffolding.

\section{Acknowledgements}

We thank Woonki Chung and Alisa Kang of the University Information Service at Georgetown University, and Michael Requa and Carl Chesal at Cycle Computing for their assistance in running Trinity and Cufflinks. Dr. Zachary Adelman provided valuable advice on running TransPS. Drs. Nathan Edwards, Shaun Brinsmade, and Martha Weiss, Mr. Zachary Batz and two anonymous reviewers provided constructive comments on this manuscript.

\section{Funding}

This work was supported in part by the National Institutes of Health (grant no. 1R15Al111328-01 to PAA) and the National Natural Science Foundation of China (grant no. 81420108024 to XGC). The funding bodies do not have a role in the design of the study and collection, analysis, and interpretation of data and in writing the manuscript. 


\section{Availability of data and materials}

The datasets supporting the conclusions of this article are available in the NCBI Short Read Archive, with the accession numbers SRA044835 and SRA051478 (embryos), SRA063587 (larvae) and PRJNA268379 (adults).

\section{Authors' contributions}

$\mathrm{XH}$ designed the study, performed the assemblies, assessed the quality of the assemblies and drafted the manuscript. XGC participated in the design of the study and the analysis of the data. PAA conceived of the study, participated in its design and coordination and helped to draft the manuscript. All authors read and approved the final manuscript.

\section{Competing interests}

The authors declare that they have no completing interests.

\section{Consent for publication}

Not applicable.

\section{Ethics approval and consent to participate}

Not applicable.

\section{Author details}

${ }^{1}$ Department of Biology, Georgetown University, 37th and O Streets NW, Washington, DC 20057, USA. ${ }^{2}$ Key Laboratory of Prevention and Control for Emerging Infectious Diseases of Guangdong Higher Institutes, Department of Pathogen Biology, School of Public Health and Tropical Medicine, Southern Medical University, Guangzhou, China.

\section{Received: 28 September 2015 Accepted: 7 July 2016}

Published online: 27 July 2016

\section{References}

1. Metzker ML. Sequencing technologies - the next generation. Nat Rev Genet. 2010;11:31-46

2. Genome 10K Project. https://genome10k.soe.ucscedu/. Accessed 7 Apr 2015.

3. i5k Genome Sequencing Initiative for Insects and Other Arthropods. http:// www.arthropodgenomes.org/wiki/i5K. Accessed 7 Apr 2015.

4. Yang HJ, Ratnapriya R, Cogliati T, Kim JW, Swaroop A. Vision from nex generation sequencing: Multi-dimensional genome-wide analysis for producing gene regulatory networks underlying retinal development, aging and disease. Prog Retin Eye Res. 2015;46:1-30

5. Elmer KR, Meyer A. Adaptation in the age of ecological genomics: insights from parallelism and convergence. Trends Ecol Evol. 2011;26:298-306.

6. Wang X, Cairns MJ. Understanding Complex Transcriptome Dynamics in Schizophrenia and Other Neurological Diseases Using RNA Sequencing. Int Rev Neurobiol. 2014:116:127-52.

7. Misof B, Liu S, Meusemann K, Peters RS, Donath A, Mayer C, et al. Phylogenomics resolves the timing and pattern of insect evolution. Science. 2014;346:763-7.

8. Ozsolak F, Milos PM. RNA sequencing: advances, challenges and opportunities. Nat Rev Genet. 2011;12:87-98

9. Wang Z, Gerstein M, Snyder M. RNA-Seq: a revolutionary tool for transcriptomics. Nat Rev Genet. 2009;10:57-63.

10. Martin JA, Wang Z. Next-generation transcriptome assembly. Nat Rev Genet. 2011;12:671-82

11. Surget-Groba Y, Montoya-Burgos II. Optimization of de novo transcriptome assembly from next-generation sequencing data. Genome Res. 2010;20:1432-40

12. Poelchau MF, Reynolds JA, Elsik CG, Denlinger DL, Armbruster PA. Deep sequencing reveals complex mechanisms of diapause preparation in the invasive mosquito, Aedes albopictus. P R Soc B. 2013;280:20130143.

13. Poelchau MF, Reynolds JA, Denlinger DL, Elsik CG, Armbruster PA. Transcriptome sequencing as a platform to elucidate molecular components of the diapause response in the Asian tiger mosquito. Physiol Entomol. 2013:38:173-81.

14. Liu M, Adelman ZN, Myles KM, Zhang L. A transcriptome post-Scaffolding method for assembling high quality contigs. Comput Biol J. 2014. doi:10.1155/2014/961823

15. Huang X, Poelchau MF, Armbruster PA. Global Transcriptional Dynamics of Diapause Induction in Non-Blood-Fed and Blood-Fed Aedes albopictus. Plos Neglect Trop D. 2015;9:e0003724
16. Trapnell C, Williams BA, Pertea G, Mortazavi A, Kwan G, van Baren MJ, et al. Transcript assembly and quantification by RNA-Seq reveals unannotated transcripts and isoform switching during cell differentiation. Nat Biotech. 2010;28:511-5.

17. Lu B, Zeng Z, Shi T. Comparative study of de novo assembly and genome-guided assembly strategies for transcriptome reconstruction based on RNA-Seq. Sci China Life Sci. 2013;56:143-55.

18. Marchant A, Mougel F, Mendonca V, Quartier M, Jacquin-Joly E, da Rosa JA, et al. Comparing de novo and reference-based transcriptome assembly strategies by applying them to the blood-sucking bug Rhodnius prolixus. Insect Biochem Molec. 2016:56:25-33.

19. Poelchau MF, Reynolds JA, Elsik CG, Denlinger DL, Armbruster PA. RNA-Seq reveals early distinctions and late convergence of gene expression between diapause and quiescence in the Asian tiger mosquito, Aedes albopictus. Jxp Biol. 2013:216:4082-90.

20. Nene V, Wortman JR, Lawson D, Haas B, Kodira C, Tu Z, et al. Genome sequence of Aedes aegypti, a major arbovirus vector. Science. 2007;316:1718-23.

21. Chen $X-G$, Jiang $X, G u J, X u M, W u Y$, Deng $Y$, et al. The genome sequence of the Asian Tiger mosquito, Aedes albopictus, reveals insights into its biology, genetics and evolution. P Natl Acad Sci USA. 2015;112:E5907-15.

22. Grabherr MG, Haas BJ, Yassour M, Levin JZ, Thompson DA, Amit I, et al. Full-length transcriptome assembly from RNA-Seq data without a reference genome. Nat Biotech. 2011;29:644-52

23. Haas BJ, Papanicolaou A, Yassour M, Grabherr M, Blood PD, Bowden J, et al. De novo transcript sequence reconstruction from RNA-seq using the Trinity platform for reference generation and analysis. Nat Protoc. 2013:8:1494-512.

24. Trapnell C, Roberts A, Goff L, Pertea G, Kim D, Kelley DR, et al. Differential gene and transcript expression analysis of RNA-seq experiments with TopHat and Cufflinks. Nature Protoc. 2012;7:562-78.

25. Camacho C, Coulouris G, Avagyan V, Ma N, Papadopoulos J, Bealer K, et al. BLAST+: architecture and applications. BMC Bioinformatics. 2009:10:421.

26. Poelchau MF, Huang X, Goff A, Reynolds J, Armbruster P. An Experimental and Bioinformatics Protocol for RNA-seq Analyses of Photoperiodic Diapause in the Asian Tiger Mosquito, Aedes albopictus. J Vis Exp. 2014;93:e51961.

27. Li WZ, Jaroszewski L, Godzik A. Clustering of highly homologous sequences to reduce the size of large protein databases. Bioinformatics. 2001;17:282-3.

28. Li W, Godzik A. Cd-hit: a fast program for clustering and comparing large sets of protein or nucleotide sequences. Bioinformatics. 2006;22:1658-9.

29. VectorBase. www.vectorbase.org. Accessed 8 Oct 2014

30. Langmead B, Salzberg SL. Fast gapped-read alignment with Bowtie 2. Nat Methods. 2012;9:357-9.

31. Waterhouse RM, Zdobnov EM, Tegenfeldt F, Li J, Kriventseva EV. OrthoDB: the hierarchical catalog of eukaryotic orthologs in 2011. Nucleic Acids Res. 2011:39 Suppl 1:D283-8.

32. Young MD, Wakefield MJ, Smyth GK, Oshlack A. Gene ontology analysis for RNA-seq: accounting for selection bias. Genome Biol. 2010;11:R14.

33. EnsemblMetazoa. metazoa.ensembl.org/biomart/martview. Accessed 23 Jun 2015

34. KEGG API. http://www.kegg.jp/kegg/rest/keggapi.html. Accessed 25 Jun 2015.

35. Bao E, Jiang T, Girke T. BRANCH: boosting RNA-Seq assemblies with partial or related genomic sequences. Bioinformatics. 2013;29:1250-9.

36. Henschel R, Lieber M, Wu L-S, Nista PM, Haas BJ, LeDuc RD. Trinity RNA-Seq assembler performance optimization. In: Proceedings of the 1st Conference of the Extreme Science and Engineering Discovery Environment: Bridging from the eXtreme to the campus and beyond. Chicago, Illinois, USA. 2012; doi: $10.1145 / 2335755.2335842$

37. Zhao QY, Wang Y, Kong YM, Luo D, Li X, Hao P. Optimizing de novo transcriptome assembly from short-read RNA-Seq data: a comparative study. BMC Bioinformatics. 2011;12 Suppl 14:S2.

38. Li B, Fillmore N, Bai Y, Collins M, Thomson JA, Stewart R, et al. Evaluation of de novo transcriptome assemblies from RNA-Seq data. Genome Biol. 2014;15:553.

39. Li B, Dewey CN. RSEM: accurate transcript quantification from RNA-Seq data with or without a reference genome. BMC Bioinformatics. 2011;12:323.

40. Bai Y, Dougherty L, Xu K. Towards an improved apple reference transcriptome using RNA-seq. Mol Genet Genomics. 2014:289:427-38.

41. Janes J, Hu F, Lewin A, Turro E. A comparative study of RNA-seq analysis strategies. Brief Bioinformat. 2015. doi:10.1093/bib/bbv007. 